\title{
Visual Cues to Restore Student Attention based on Eye Gaze Drift, and Application to an Offshore Training System
}

\author{
Andrew Yoshimura* \\ Univ. of Louisiana at Lafayette \\ Lafayette, Louisiana \\ andrewyosh@yahoo.com
}

\author{
Adil Khokhar \\ Univ. of Louisiana at Lafayette \\ Lafayette, Louisiana \\ guvadilk@gmail.com
}

\author{
Christoph W Borst \\ Univ. of Louisiana at Lafayette \\ Lafayette, Louisiana \\ cwborst@gmail.com
}

\begin{abstract}
Drifting student attention is a common problem in educational environments. We demonstrate 8 attention-restoring visual cues for display when eye tracking detects that student attention shifts away from critical objects. These cues include novel aspects and variations of standard cues that performed well in prior work on visual guidance. Our cues are integrated into an offshore training system on an oil rig. While students participate in training on the oil rig, we can compare our various cues in terms of performance and student preference, while also observing the impact of eye tracking. We demonstrate experiment software with which users can compare various cues and tune selected parameters for visual quality and effectiveness.
\end{abstract}

\section{CCS CONCEPTS}

\section{- Human-centered computing $\rightarrow$ Virtual reality.}

\section{KEYWORDS}

Educational VR; Attention; Eye Tracking; Visual Cues

\section{ACM Reference Format:}

Andrew Yoshimura, Adil Khokhar, and Christoph W Borst. 2019. Visual Cues to Restore Student Attention based on Eye Gaze Drift, and Application to an Offshore Training System. In Symposium on Spatial User Interaction (SUI '19), October 19-20, 2019, New Orleans, LA, USA. ACM, New York, NY, USA, 2 pages. https://doi.org/10.1145/3357251.3360007

\section{INTRODUCTION}

We are presenting 8 attention-restoring visual cues to encourage students to return visual focus to the correct object when their attention shifts elsewhere. As a simple example, we measure inattention by using the gaze angle between the student's eye gaze vector and the vector from the student to the target object. Cues are activated once the gaze angle surpasses a threshold. We have described a more general inattention score elsewhere [4]. Our cues are specifically being used to "restore attention" after it is lost. We believe this will result in a more subtle and effective use of the cues, when compared to previous works [7] in which visual cues are used to "guide attention" from the start. In addition to adapting some of

Permission to make digital or hard copies of part or all of this work for personal or classroom use is granted without fee provided that copies are not made or distributed for profit or commercial advantage and that copies bear this notice and the full citation on the first page. Copyrights for third-party components of this work must be honored

For all other uses, contact the owner/author(s).

SUI '19, October 19-20, 2019, New Orleans, LA, USA

(c) 2019 Copyright held by the owner/author(s).

ACM ISBN 978-1-4503-6975-6/19/10.

https://doi.org/10.1145/3357251.3360007 the best-performing cues from prior work, we include a few of our own cue variations for future assessment.

Each one of our cues has been integrated into an offshore training system on a virtual oil rig (Fig. 1k) in order to gather data about cue performance (time and head/eye movements necessary to restore student attention) and the benefits of eye tracking. Students will be tasked with learning about various features of the oil rig. Our cues will be used to assist the student when inattention is detected.

Once our cues have been compared and optimized, they will be integrated with a pedagogical agent that presents content in a VR field trip [4]. This will promote student attention and provide a more engaging learning environment in educational VR.

\section{ATTENTION-RESTORING CUES}

\subsection{Preliminary Details}

Audience members will be able to cycle through cues and toggle eyetracking on and off. They will also be able to manipulate multiple parameters, which allow control over features of the cues.

Each cue shares two common parameters: transparency/fade strength and cue size.

Some cues have a special placement parameter that determines whether they are placed along an arc on a head centered sphere with radius being the distance between the head and the target or along a cubic Hermite curve like the one described by Biocca et al. [1].

In order to handle objects in the VR scene occluding our cues, select cues are rendered such that they appear through other objects.

\subsection{Cue Descriptions}

The standard arrow (Fig. 1a) is a single 3D arrow. It is placed along the arc above and is oriented to point towards the target. A positioning parameter allows for the arrow to be offset some percentage towards the target.

The trail of arrows (Fig. 1b/1c) places multiple arrows along the arc or curve between the student's gaze and the target object. This is expected to provide a stronger cue than a single arrow.

The navigation sphere (Fig. 1d) is a novel cue that is a field of arrows on a head-centered sphere. Arrow placement does not depend on any gaze tracking, so arrows appear static rather than moving. The arrows appear at vertices of an (invisible) icosphere and are oriented to point along an arc towards the target, as for the standard arrow. A parameter varies the number of arrows, corresponding to different icospheres with different levels of detail.

The attention funnel (Fig. 1e) is based on the one created by Biocca et al., which was created by placing multiple rings along a 


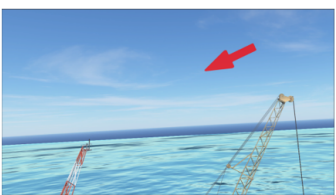

a) Arrow

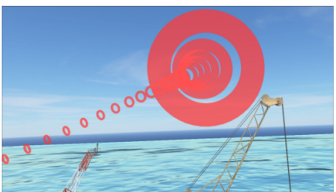

e) Attention Funnel

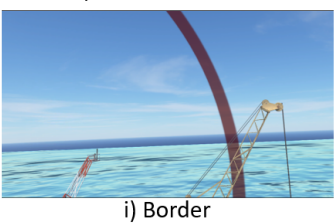

i) Border

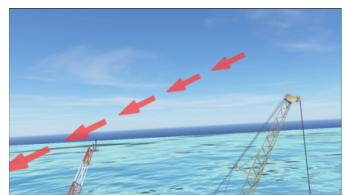

b) Trail of Arrows Along Arc

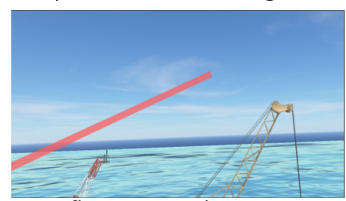

f) Line Strip Along Arc

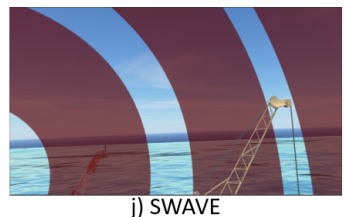

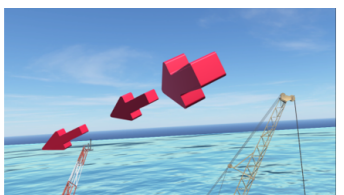

c) Trail of Arrows Along Curve

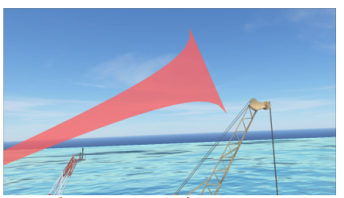

g) Line Strip Along Curve

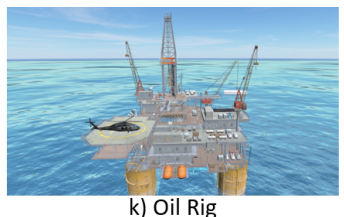

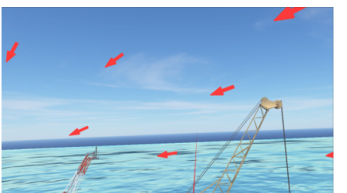

d) Navigation Sphere

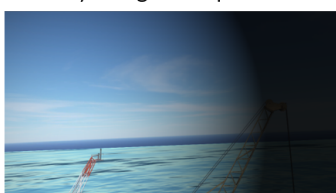

h) Tunnel Vision

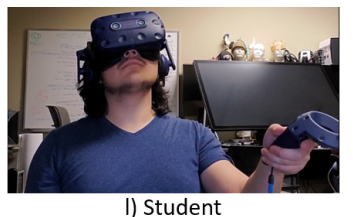

Figure 1: Attention-Restoring Cues Posed Towards and Offscreen Target Object (a-j), Offshore Oil Rig (k), Student interacting with the demonstration (1)

cubic curve between the student and the target [1]. A rendering parameter changes the rendering style (ring or goalpost).

The line strip (Fig. 1f/1g) is a simple cue that shares features with both Wedge [3] and 3DPath [5]. A thick line strip (appearing like a curved banner) is displayed along the arc or cubic curve, showing a full path from the student's current gaze to the target. Contrasting with 3DPath, the line strip is rendered to show through any occluding objects.

Our tunnel vision (Fig. $1 \mathrm{~h}$ ) cue is a vignette-type cue, very similar to Fade to Black [2], that progressively darkens portions of the scene far from the target as the student attention drifts. We use a screenspace shader to orient a tunnel effect in front of the student (with an offset toward the target), which gets more prominent (darker, sharper) the farther the student looks away. The open portion of the tunnel never leaves the student's viewport completely, to prevent confusion about where to shift visual focus. A parameter controls how dark the tunnel gets.

The border (Fig. 1i) displays a circle on a head-centered sphere, where the circle's position on the sphere is centered around the target object. The circle's edge follows the student's gaze until it reaches the boundary. The boundary is a parameter that describes how far the border will move away from the target object. A movement parameter then decides how the border will move when it reaches the boundary (static position or oscillate between gaze and boundary). This cue is similar to Halo [3], however, we do not experience clutter as there is only one target object at a time.

The DynSWAVE (Fig. 1j) is based on the one created by Renner and Pfeiffer, in which concentric circles move along a head centered sphere towards the target object, with the speed controlled by the student's eye gaze angle (the circles move faster as the student looks away from the target) [6]. A parameter allows for the user to scale the speed of the circles. This cue performed well in the Renner study, giving us good reason to compare it with our others.

\section{CONCLUSIONS}

We present 8 attention-restoring visual cues to encourage student attention when eye tracking detects a drift. Our demonstration software will allow us to gather data about subjective preference for cues to provide a more fluid and engaging learning experience in educational VR.

\section{ACKNOWLEDGMENTS}

This material is based upon work supported by the National Science Foundation under Grant No. 1815976 and by the Louisiana Board of Regents under contract LEQSF(2019-20)-ENH-DE-22

\section{REFERENCES}

[1] Frank Biocca, Arthur Tang, Charles Owen, and Fan Xiao. 2006. Attention funnel: Omnidirectional 3D Cursor for Mobile Augmented Reality Platforms. Proceedings of the SIGCHI conference on Human Factors in computing systems - CHI 06 (2006). https://doi.org/10.1145/1124772.1124939

[2] Fabien Danieau, Antoine Guillo, and Renaud Dore. 2017. Attention guidance for immersive video content in head-mounted displays. 2017 IEEE Virtual Reality (VR) (2017). https://doi.org/10.1109/vr.2017.7892248

[3] Uwe Gruenefeld, Abdallah El Ali, Susanne Boll, and Wilko Heuten. 2018. Beyond Halo and Wedge. Proceedings of the 20th International Conference on HumanComputer Interaction with Mobile Devices and Services - MobileHCI 18 (2018). https: //doi.org/10.1145/3229434.3229438

[4] Adil Khokhar, Andrew Yoshimura, and Christoph W. Borst. 2019. Pedagogical Agent Responsive to Eye Tracking in Educational VR. 2019 IEEE Conference on Virtual Reality and 3D User Interfaces (VR) (2019). https://doi.org/10.1109/vr.2019. 8797896

[5] Patrick Renner, Jonas Blattgerste, and Thies Pfeiffer. 2018. A Path-Based Attention Guiding Technique for Assembly Environments with Target Occlusions. 2018 IEEE Conference on Virtual Reality and 3D User Interfaces (VR) (2018). https: //doi.org/10.1109/vr.2018.8446127

[6] Patrick Renner and Thies Pfeiffer. 2017. Attention guiding techniques using peripheral vision and eye tracking for feedback in augmented-reality-based assistance systems. 2017 IEEE Symposium on 3D User Interfaces (3DUI) (2017). https://doi.org/10.1109/3dui.2017.7893338

[7] Sylvia Rothe, Daniel Buschek, and Heinrich Hußmann. 2019. Guidance in Cinematic Virtual Reality-Taxonomy, Research Status and Challenges. Multimodal Technologies and Interaction 3, 1 (2019), 19. https://doi.org/10.3390/mti3010019 